\title{
Current Perspective of High-Intensity Focused Ultrasound for Benign Gynecological Tumor: A Review
}

\author{
Randy Polyanna Po ${ }^{1}$ and AixingziAilii, ${ }^{1,2 *}$ \\ ${ }^{1}$ School of Medicine, Tongji University, PR China \\ ${ }^{2}$ Department of Gynecology, Shanghai First Maternity Hospital affiliated to Tongji University, PR China
}

Submission: April 23, 2017; Published: June 01, 2017

*Corresponding author: Aixingzi Aili, Department of Gynecology, Shanghai First Maternity Hospital affiliated to Tongji University, Shanghai 200126, PR China, Tel: +8618918760250; Email: 1819483078@qq.com

Abstract

High intensity focused ultrasound (HIFU) has been used extensively in various medical fields. The outcome of treatments have been promising and been proven to be effective and safe. In gynecological disease, HIFU has been used for various diseases such as uterine fibroid, adenomyosis, polycystic ovarian syndrome and even uterine scar pregnancy. This review is written due to increased use of HIFU as an effective and safe treatment of benign gynecological tumor worldwide. It includes, the use of HIFU in treating uterine fibroid and adenomyosis, advantages and disadvantages of HIFU, the treatment outcomes and safety of HIFU.

Keywords: High intensity focused ultrasound; Adenomyosis; Uterine fibroid

\section{Introduction}

In 1942 Lynn [1] was the first one who did the experiments on living animals using medium or high intensity of ultrasound. The result of the experiment showed that this high intensity ultrasound was able to induce necrosis in targeted tissue without damaging the surrounding vital structures. However it was not until 1958 when the first therapeutic application of HIFU was used in the field of neurosurgery, precisely neurosurgical treatment of movement disorders, neuropathic pain and hypersensitivity [2]. Currently, HIFU is clinically used in other diseases such as prostate cancer, uterine fibroids, uterine adenomyosis and bone cancer [3].

The procedure of HIFU is relatively simple and convenient as compared to other treatment options. Patients are fully examined and MR imaging is ordered to decide their eligibility and treatment plan prior to the treatment. Bowel preparation is also a vital preparation prior the treatment. Patients lie on prone position and water bath or gel pad is placed over the transducer. Skin is then prepared by removing hair, dirt and evaluated if the patients have scar between umbilicus and pubic bone. A urinary catheter is inserted into the bladder filled with sterile saline ensured bladder volume. Intravenous sedation is then administrated using fentanyl and midozolam for analgesic.
A treatment plan is then calculated by calculating energy, location, and number of ultrasound sonications, or energy bursts, needed to treat the focal volume. A narrow cylindrical shape multiple of high intensity energy called sonications is then released. The sum of the single sonications must cover the entire focal volume. Typically, the sonications employed are short and multiple (60-90 sonications lasting $20 \mathrm{~s}$ each; $1.000-7.000$ joules of energy each one) with continual thermal feedback [4].

\section{HIFU for benign gynecological tumor}

HIFU is presently used in gynecological diseases such as, uterine fibroid, adenomyosis and in the treatment of uterine scar pregnancy. The use of HIFU for gynecological tumors have been used extensively in USA, Europe and Asia. While The ExAblate device (Insightec, Tirat Carmel, Israel) is currently the only United States Food and Drug Administration (FDA)-approved MR-guided HIFU system. Asian and European countries mostly use the The JC HIFU system (Chongqing Haifu Technology, Chongqing, China). In China, HIFU for benign gynecological tumor such as uterine fibroids and adenomyosis has been applied and became the first-line therapy in many clinical centers $[5,6]$.

Uterine fibroids are also known as leimyoma is benign tumors that arise from smooth muscle cells of uterine myometrium. It is 
also the most common tumors of the female reproductive system, occurring in atleast $20 \%$ to $25 \%$ of all reproductive-age women [7]. While half of women with uterine fibroids are asymptomatic, it can also significantly affect morbidity and women's quality of life. Symptoms can vary depends on size and location, but mostly can cause abnormal uterine bleeding, heavy menstrual bleeding, pelvic pain, pressure and infertility. The choices of treatment of uterine fibroids are available extensively, from conservative treatment to surgical treatment, the choice mostly depends on size, number, location, age and whether or not the women is expecting child bearing. Conservative treatments include hormonal therapy, uterine artery embolization (UAE) and HIFU [8]. The principle of HIFU in the treatment of uterine fibroids is the induction of focused ultrasonic energy that causes coagulation necrosis of a target lesion but does not damage the surrounding tissue [9].

Adenomoysis is another benign gynecological tumor that affects women during their reproductive age and its incidence is gradually increasing [10]. Like uterine fibroids, adenomyosis also influence women's quality of life with symptoms such as secondary dysmenorrhea, menorrhagia and subfertility [11]. Although the first line of treatment for dysmenorrhea in adenomyosis is hormonal contraceptive [12], this choice is only a temporary treatment. Hysterectomy is the sole radical permanent cure, however this option may not be the most preferred option for patients with fertility desires and those who are unwilling to resect their uteri.

\section{Advantages and disadvantages of HIFU}

HIFU as a non-invasive treatment for benign gynecological tumor has more advantages as compare to other treatment options. As a conservative treatment, HIFU has the benefit of sparing the uterus. Most women are not willing to undergo hysterectomy as it might make them feel less women. Especially women that have fertility desire, HIFU does not require patients to delay their pregnancy longer than other treatment options. While the suggested time to conception after the treatment is 6-12 months, a study was done in 51 women and the mean time to conception was 8 months [7].

HIFU also has the advantage of fast recovery time which enable patients to go back to their daily activities just one day after the procedure [13]. HIFU is also cost effective compared to other treatment options. Although no study has yet been done to compare to cost effectiveness.

While HIFU can be beneficial to other procedures, HIFU also has some technical drawbacks and limitations. One of the most challenging disadvantages is the time of treatment. As discussed before, the principle of HIFU is by applying ultrasound energy to the lesion area. The energy is applied by a single sonication spots to target the lesion while the sonication area is small many sonication areas are required. Moreover, during the application of energy, normal tissue surrounding the lesion can absorb some of the energy. To avoid damage to the normal tissue, a break period is needed to cool down the thermal build up. This process adds up to the longer treatment time, a medium size $50-100 \mathrm{~cm} 3$ uterine fibroid might take several hours to complete. While during the procedure patients are not allowed to freely move and have to stay still lying, some patients might feel uncomfortable. Medical personnel also have to be there during the procedure [14].

Other disadvantages is that some lesion might not visible due to obstruction of other tissues such as bone, gas or intestines. This problem causes segments of bowel anterior to uterine fibroids to become a contraindication for treatment. As gas might reflect back the energy to the transducer and lead to unwanted heating and burns [14]. The areas surrounding the location of the treatment like the intestines could be affected if the intestines absorb or accidentally damaged by the ultrasound waves, this might cause intestinal problems.

\section{Results Outcome}

Studies of the effectiveness of HIFU have been done in many countries across Asia and Europe, and the results were promising. The studies tackled uterine fibroids from different angles such as the symptoms severity scale (SSS), uterine fibroid symptoms and quality of life(UFS-QOL), changes in volume of the lesion size. While as the prominent symptoms of adenomyosis is dysmenorrhea, most studies compare the changes in dysmenorrhea by using the visual analog system (VAS).

Funaki et al. [15] conducted a study of 91 uterine fibroid patients type 1, 2 and 3 with 24-months follow up on the patients. The changes of volume ratio were satisfying in type 1 and 2 uterine fibroid patients with changes from $36.5 \% 6$ months after the procedure to $39.5 \% 24$ months after the procedure. Unfortunately, type 3 uterine fibroid did not show significant changes in volume ratio but smaller volume were noted after 12 and 24 months after the procedure. While the SSS analysis was satisfying enough in type 1 and 2 uterine fibroid, initially the mean SSS score was $35.1 \pm 21.0$, and the SSS dropped significantly 3 months after the treatment and still remain low 24 months after the treatment.

Another study was done in Korea to understand the effectiveness of HIFU both in uterine fibroid and adenomoysis by Lee et al. [16]. With a total of 618 patients, 272 were diagnosed with uterine fibroid and 346 were diagnosed with adenomoysis. The reduction rate were even higher than the study done by Funaki et al. [15] in Japan, 58.08\%, 66.18\% and $77.59 \%$ at 3,6 and 12 months after the treatment. While the SSS reduction rate were $55.58,52.76 \%$ and $50.39 \%$ by 3,6 and 12 months respectively. This study also includes the UFS-QOL for both uterine fibroid and adenomyosis, and the result shows that the UFS-QOL for uterine fibroid increasing rate were $42.66 \%$ after 3 months, $43.50 \%$ after 6 months and $43.45 \%$ after 12 months. For adenomyosis the result of volume reduction rate was also promising, with $43.99 \%$ after 3 months, $47.01 \%$ after 6 months and $53.89 \%$ after 12 months. 
Studies were done in Chongqing China to see the efficacy of HIFU for adenomyosis by Shui et al. [5] and Zhang et al. [17]. Both studies also showed promising results. Shui et al. [5] did a two-year follow up study to learn about the changes in dysmenorrhea degree before and after the treatment. The relief rate was $84.7 \%$ after 3 months, $84.7 \%$ after 1 year and $82.3 \%$ after 2 years. While Zhang et al. [17] studied dysmenorrhea by using a 5-point categoric scale (1, not affected; 2 , a little affected; 3 , somewhat affected; 4 , greatly affected; 5 , very greatly affected) and then categorized the results into complete relief, partial relief, minor relief or not effective depending on the changes in the score. The results show $87.9 \%$ and $81.9 \%$ of complete relief for focal adenomyosis and diffuse adenomyosis respectively. Another study by Gong et al. [18] compared the efficacy of HIFU in treatment of adenomoysis through hypotense and isotense lesions and the result show that there were no significant difference between the groups.

\section{Safety Evaluation}

As an effective treatment for uterine fibroids and adenomyosis, a lot of studies have also been done to see the safety of HIFU. A study was done in Chongqing by Chen et al. [19] by reviewing 9988 cases of uterine fibroids and adenomyosis after treatment by ultrasound-guided ultrasound ablation. The study shows that $10.6 \%$ of the patients presented with 1305 events of adverse reactions. The study uses the SIR classification system in which $1228(94.1 \%)$ of these reactions were graded as Class A; $45(3.4 \%)$ as Class B; 24 as (1.8\%) Class C and $8(0.6 \%)$ as Class D. With the most frequent adverse reaction to be brown color vaginal secretion that lasted between 3 to 14 days. Adverse reaction that occurred immediately after the treatment was lower abdominal pain or buttock pain, which can be relieved by taking pain killer if necessary. Other common reverse reaction was abdominal skin burn in which 2 patients was treated with surgical removal of necrotic skin. The study also emphasizes that no permanent or fatal injury has been reported.

Another study was done to see the complication that occur during the therapy and after the therapy by Feng et al. [20]. During the procedure, $92.3 \%$ of the patients experience adverse effects such as lower limb radiation pain, the sacral tail pain, skin-burning pain, target region pain, groin pain or hip discomfort. While after the treatment $87.1 \%$ of the patients have lower abdominal pain complain, other discomfort reported were vaginal discharge and skin burn but no major complaint was received from all 417 patients during their follow ups.

One of the main procedures to assess whether HIFU is applicable for the patients is through contrast- enhanced ultrasound. Low level of ultrasound contrast agent is relatively safe, however there were cases of death reported [21]. Cheng et al. [22] did a study to compare the adverse effects of HIFU by diving the patients into two groups, contrast-enhanced ultrasound and the other group without. The results show that the contrast agent may increase the incidences of some common
HIFU-related adverse effects during HIFU treatment but most of which were acceptable and self-limited. It is noted that the patients' history should be understood carefully if the patients have history of hypertension or taking nonsteroidal antiinflammatory drugs.

\section{Pregnancy after HIFU}

Successful pregnancies HIFU have been reported in many studies [7,23]. One of the studies by Rabinovici et al. [7], show that there were 54 pregnancies out of 51 patients after HIFU procedure for treatment of uterine fibroid. Live births occurred in $41 \%$ of pregnancies, with a $28 \%$ spontaneous abortion rate, an $11 \%$ rate of elective pregnancy termination, and $11(20 \%)$ ongoing pregnancies beyond 20 gestational weeks. The mean birth weight was $3.3 \mathrm{~kg}$, and the vaginal delivery rate was $64 \%$. While another study in China by Zhou et al. [23] show that 21 out of 44 patients were able to deliver healthy babies without uterine rupture.

\section{Conclusion}

HIFU is a non-invasive, effective and safe treatment choice for benign gynecological tumor. Although some adverse reactions were reported, with a unified and standardized protocol the adverse reactions can be monitored and lessen significantly. With advantages that outweigh the disadvantages we concluded that HIFU is a promising and safe procedure for benign gynecological tumor. However as a relatively new technique for gynecological diseases, more studies need to be conducted.

\section{Declaration of Conflicting Interests}

The author declared no potential conflicts of interest with respect to the research, authorship, and/or publication of this article.

\section{Funding}

The author received no financial support for the research, authorship, and/or publication of this article.

\section{References}

1. Miller AE (1942) From the Departments of Neurology and of Anatomy, College of Physicians and Surgeons. Columbia University, New York, USA, p. 2.

2. Foley J, Eames M, Snell JW (2014) Image-guided focused ultrasound: State of the technology and the challenges that lie ahead Image-guided focused ultrasound: state of the technology and the challenges that lie ahead.

3. Masciocchi C, Conchiglia A, Gregori LM, Arrigoni F, Zugaro L, et al. (2014) Critical role of HIFU in musculoskeletal interventions. Radiol Medica 119(7): 470-475.

4. Mahmoud MZ, Alkhorayef M, Alzimami KS, Aljuhani MS, Sulieman A (2014) High-Intensity Focused Ultrasound (HIFU) in Uterine Fibroid Treatment: Review Study. Pol J Radiol 79: 384-390.

5. Shui L, Mao S, Wu Q Huang G, Wang J, et al. (2015) High-intensity focused ultrasound (HIFU) for adenomyosis: Two-year follow-up results. Ultrason Sonochem 27: 677-681.

6. Zhao WP, Chen JY, Zhang L, Li Q, Qin J, et al. (2013) Feasibility of ultrasound-guided high intensity focused ultrasound ablating uterine 
fibroids with hyperintense on T2-weighted MR imaging. Eur J Radiol 82(1): e43-e49.

7. Rabinovici J, David M, Fukunishi H, Morita Y, Gostout BS, et al. (2010) Pregnancy outcome after magnetic resonance-guided focused ultrasound surgery (MRgFUS) for conservative treatment of uterine fibroids. Fertil Steril 93(1): 199-209.

8. Masciocchi C, Arrigoni F, Ferrari F, Victor A, Iafrate S, et al. (2017) Uterine fibroid therapy using interventional radiology mini-invasive treatments : current perspective. Med Oncol 34(4): 1-12.

9. Wang F, Tang L, Wang L, Wang X, Chen J, et al. (2014) Ultrasound-Guided High-Intensity Focused Ultrasound vs Laparoscopic Myomectomy for Symptomatic Uterine Myomas. J Minim Invasive Gynecol 21(2): 279284

10. Levy G, Dehaene A, Laurent N, Lernout M, Collinet P, et al. (2013) An update on adenomyosis. Diagn Interv Imaging 94(1): 3-25.

11. Peric H, Fraser IS (2006) The symptomatology of adenomyosis. Best Pract Res Clin Obstet Gynaecol 20(4): 547-555.

12. Priya K, Rajaram S, Goel N (2016) European Journal of Obstetrics \& Gynecology and Reproductive Biology Comparison of combined hormonal vaginal ring and low dose combined oral hormonal pill for the treatment of idiopathic chronic pelvic pain : a randomised trial \$. Eur J Obstet Gynecol 207: 141-146.

13. Shehata IA (2012) Treatment with high intensity focused ultrasound: Secrets revealed. Eur J Radiol 81(3): 534-541.

14. Schlesinger D1, Benedict S, Diederich C, Gedroyc W, Klibanov A, et al (2013) MR-guided focused ultrasound surgery, present and future. Am Assoc Phys Med 40(8): 080901

15. Funaki K, Fukunishi H, Sawada K (2009) Clinical outcomes of magnetic resonance-guided focused ultrasound surgery for uterine myomas: 24-Month follow-up. Ultrasound Obstet Gynecol 34(5): 584-589.
16. Lee JS, Hong GY, Park BJ, Kim TE (2015) Ultrasound-guided highintensity focused ultrasound treatment for uterine fibroid \& adenomyosis: A single center experience from the Republic of Korea. Ultrason Sonochem 27: 682-687.

17. Zhang X, Li K, Xie B, He M, He J, et al. (2014) Effective ablation therapy of adenomyosis with ultrasound-guided high-intensity focused ultrasound. Int J Gynecol Obstet 124(3): 207-211.

18. Gong C, Setzen R, Liu Z, Liu Y, Xie B, et al. (2017) High intensity focused ultrasound treatment of adenomyosis: The relationship between the features of magnetic resonance imaging on T2 weighted images and the therapeutic efficacy. Eur J Radiol 89: 117-122.

19. Chen J, Chen W, Zhang L, Li K, Peng S, et al. (2015) Safety of ultrasoundguided ultrasound ablation for uterine fibroids and adenomyosis: A review of 9988 cases. Ultrason Sonochem 27: 671-676.

20. Feng Y, Hu L, Chen W, Zhang R, Wang X, et al. (2017) Safety of ultrasoundguided high-intensity focused ultrasound ablation for diffuse adenomyosis: A retrospective cohort study. Ultrason-Sonochemistry 36: 139-145.

21. Van Camp G, Droogmans S, Cosyns B (2007) Bio-effects of ultrasound contrast agents in daily clinical practice: Fact or fiction? Eur Heart J 28(10): 1190-1192.

22. Cheng CQ, Zhang RT, Xiong Y, Chen L, Wang J, et al. (2015) ContrastEnhanced Ultrasound for Evaluation of High-Intensity Focused Ultrasound Treatment of Benign Uterine Diseases. Medicine Baltimore 94(16): e729.

23. Zhou CY, Xu XJ, He J (2016) Pregnancy outcomes and symptom improvement of patients with adenomyosis treated with high intensity focused ultrasound ablation. Zhonghua Fu Chan Ke Za Zhi 51(11): 845849.

\section{Your next submission with Juniper Publishers} will reach you the below assets

- Quality Editorial service

- Swift Peer Review

- Reprints availability

- E-prints Service

- Manuscript Podcast for convenient understanding

- Global attainment for your research

- Manuscript accessibility in different formats

( Pdf, E-pub, Full Text, Audio)

- Unceasing customer service

Track the below URL for one-step submission https://juniperpublishers.com/online-submission.php 